\title{
Accuracy of Healthcare Providers' Perception of Chest Compression Depth and Chest Recoil
}

\author{
Sendoa Ballesteros-Peña ${ }^{1,2,3, *}$, Gorka Vallejo-De la $\mathrm{Hoz}^{2,3,4}$, \\ Irrintzi Fernández-Aedo ${ }^{2,3}$, Alejandro Etayo-Sancho ${ }^{5}$, \\ Leire Berasaluze-Sanz ${ }^{2,4}$, Josune Domínguez-García²
}

\author{
${ }^{1}$ Integrated Healthcare Organisation \\ Bilbao-Basurto, Bilbao, Spain \\ ${ }^{2}$ Biocruces Bizkaia Health Research \\ Institute, Barakaldo, Spain \\ ${ }^{3}$ University of the Basque Country \\ (UPV/EHU), Spain \\ ${ }^{4}$ Barrualde-Galdakao Integrated \\ Healthcare Organisation, Galdakao, \\ Spain \\ ${ }^{5}$ Urduliz Advanced Life Support Unit, \\ Urduliz, Spain

\section{*Correspondence} \\ sendoa.ballesteros@ehu.eus \\ (Sendoa Ballesteros-Peña)
}

\begin{abstract}
Objective: The objective of this paper was to evaluate the validity and accuracy of healthcare providers' perception of chest compression depth and chest recoil during cardiopulmonary resuscitation (CPR). Methods: A clinical simulation study was performed with healthcare providers trained in CPR including physicians, nurses, and Emergency Medical Technicians (EMT). Following 2 minutes of hands-only-CPR on a sensor-programmed manikin, providers were able to respond to subjective questions assessing their adequacy of CPR. The providers' perception contrasted with the objective data obtained from the manikin. The validity and accuracy of CPR providers' perception of chest compression depth and chest recoil was assessed by the calculation of sensitivity, specificity and predictive values. Results: 180 Advanced or basic life support certified healthcare providers were enrolled. The degree of correlation between self-perception and actual performance was $52.2 \%$ in the thoracic compression depth and $61.7 \%$ in the chest recoil. Caregivers' perception of chest compression depth had a sensitivity of $29.4 \%$ and a specificity of $87.3 \%$. Caregivers' perception of chest recoil had a sensitivity of $30.3 \%$ and a specificity of $79.8 \%$. Conclusions: Healthcare providers' perception for evaluating the accuracy of thoracic compressions is not as accurate as objective feedback methods during CPR. This may impact patient outcomes during a cardiac arrest.
\end{abstract}

\section{Keywords}

Cardiopulmonary resuscitation, Heart massage, Cardiac arrest, Simulation

\section{Introduction}

The quality of chest compressions during CPR affects both the short-term and long-term survival after cardiac arrest. Well-performed CPR consists of good quality chest compressions with adherence to rate, depth and complete wall recoil. According to current guidelines, to be considered of high quality, chest compressions should be performed in the centre of the thorax at a rate of $100-120$ compressions per minute and at a depth of $5-6 \mathrm{~cm}$, allowing for a full chest recoil after each compression [1].

High-quality CPR maximizes the survival odds of patients with a cardiac arrest [2]. The monitoring of the quality of compressions in real time is not always possible during a cardiac arrest. Therefore, the quality of compres- sions typically depends on the providers' perception.

The objective of this paper was to evaluate the validity and accuracy of healthcare providers' perception of chest compression depth and chest recoil during cardiopulmonary resuscitation (CPR).

\section{Materials and methods}

A clinical simulation study of CPR during a cardiac arrest was performed. Critical care providers (physicians, nurses, and Emergency Medical Technicians) from Emergency Department, Emergency Medical Service or Intensive Care Units trained in CPR (BLS/ACLS certified) were included on a volunteer basis.

The sample was selected with convenience criteria, mak- 


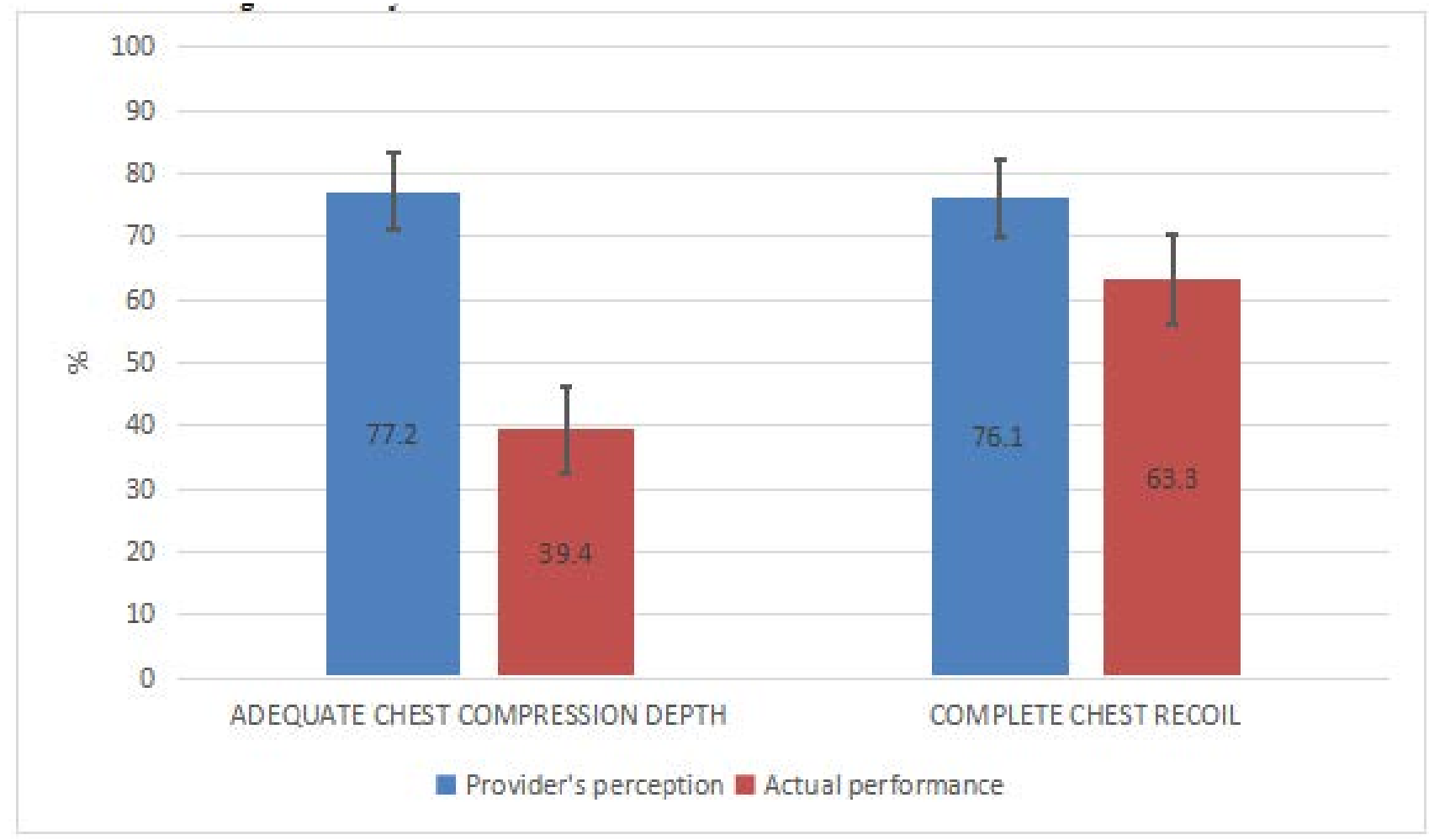

F I G U R E 1. Comparison between caregivers' perception and actual performance of chest compression and chest recoil during hands-only-CPR.

$95 \%$ confidence interval error bar.

ing a public invitation to participate among the professionals of different health centres. Professionals whose physical condition was not compatible with exercise were excluded (e.g., pregnant women). All participants were informed about the objectives of the study and they gave their written informed consent. This study was approved by the Ethics Committee of the Hospital of Basurto. The minimum sample size was estimated for a $95 \%$ confidence level and an accuracy of $7.5 \%(\mathrm{p}=\mathrm{q}=0.5)$.

All participants self-reported demographic and anthropometric data upon enrolment. Then, they were instructed to deliver hands-only-CPR on a Resusci Anne ${ }^{\circledR}$ Q-CPR manikin with SkillReporter $\left(\right.$ Laerdal $\left.{ }^{\circledR}\right)$ placed on the floor. The simulation time was set at 2 minutes based on current guidelines. Chest compression depth and residual leaning force were recorded for each participant. Compression rate was controlled with a metronome at 110 bits per minute.

Upon completion (and without the participant knowing of the results of the test), the subjects were asked to answer (Yes/No) to the following questions: "Do you believe, in general, that you have managed to press the thorax between 5 and $6 \mathrm{~cm}$ in each compression?" and "Do you believe, in general, that you have allowed a complete chest recoil after each compression?".

Chest compressions were considered adequate when the average depth during the CPR cycle was between 5 and 6 $\mathrm{cm}$. The chest recoil was considered complete when the average residual leaning force on the chest wall was $\leq$ $2.5 \mathrm{~kg}$, according to the quality parameters of the European
Resuscitation Council ${ }^{1}$.

Continuous variables are presented as means with their standard deviation (SD) and range and percentages with their $95 \%$ confidence intervals $(95 \% \mathrm{CI})$. The proportion of participants with positive and negative perception of chest compression depth and chest recoil and the actual performance results (measured by a sensor-programmedmanikin) was compared using the chi-square test. Excel 2019 (Microsoft) and SPSS 25 statistics software (IBM) were used for all statistical analyses. A $p$ value $<0.05$ was considered statistically significant.

The validity and accuracy of CPR providers' perception of chest compression depth and chest recoil was assessed by the calculation of sensitivity, specificity and predictive values for the subjective assessment versus the objective manikin data.

\section{Results}

The study consisted of 180 volunteers (124 nurses, 35 doctors and 21 Emergency Medical Technicians). 79.4\% were women and $39.4 \%$ had previously participated in more than $10 \mathrm{CPR}$ events. Each participant held a current certification in intermediate or advanced life support for healthcare professionals. The characteristics of participants in the study are summarised in Table 1.

All participants completed the 2 minutes hands-onlyCPR simulation. Based on the questionnaire, $77.2 \%$ $(95 \%$ CI $70.4-83.1)$ of the healthcare professionals 
TA B L E 1. Characteristics of the participants in the study $(n=180)$.

\begin{tabular}{|lcc|} 
& Mean (SD) & Range \\
\hline Age (years) & $38(9.7)$ & $22-65$ \\
\hline Work experience in ED or Critical Care setting (years) & $12.4(9)$ & $1-40$ \\
\hline Weight (kg) & $66.9(15.8)$ & $43.9-149$ \\
Height (cm) & $166.5(8.5)$ & $150-197$ \\
Body Mass Index $\left(\mathbf{k g} / \mathbf{m}^{2}\right)$ & $24(4.7)$ & $17.1-49.8$ \\
\hline
\end{tabular}

ED: Emergency Department; SD: Standard deviation.

TA B L E 2. Validity and predictive values of cardiopulmonary resuscitation providers' perception of chest compression depth and chest recoil.

\begin{tabular}{|c|c|c|}
\hline & Provider's perception of chest compression & Provider's perception of chest \\
\hline & depth adequacy \% $(95 \% \mathrm{CI})$ & recoil adequacy $\%(95 \% \mathrm{CI})$ \\
\hline Percentage of success & $52.2(44.7-59.7)$ & $61.7(54.1-68.7)$ \\
\hline Sensitivity & $29.4(21.2-39)$ & $30.3(19.9-43)$ \\
\hline Specificity & $87.3(76.8-93.7)$ & $79.8(71.1-86.5)$ \\
\hline Positive predictive value & $78.1(62-88.9)$ & $46.5(31.5-62.2)$ \\
\hline Negative predictive value & $44.6(36.6-53.3)$ & $66.4(57.8-74.1)$ \\
\hline
\end{tabular}

95\% CI: 95\% confidence interval.

believed their compression depth was adequate, while the objective measure demonstrated only $39.4 \%$ achieved adequate compression depth $(95 \%$ CI $32.3-47 ; \mathrm{p}<$ $0.001)$. In terms of the chest recoil metric, on the contrary, there was no statistically significant difference $(\mathrm{p}=0.125)$ between the subjective $(76.1 \%$; $95 \%$ CI $69.2-82.1)$ and objective $(63.3 \%$; $95 \%$ CI $55.8-70.4)$ measures.

The percentage of agreement between self-perception and actual performance was $52.2 \%(95 \% \mathrm{CI} 44.7-59.7)$ in the chest compression depth and $61.7 \%(95 \% \mathrm{CI} 54.1-68.7)$ in the chest recoil (Fig. 1).

The probability that the provider inaccurately assessed their compression depth or incomplete chest recoil (sensitivity) was $29.4 \%(95 \% \mathrm{CI} 21.2-39)$ and $30.3 \%(95 \% \mathrm{CI}$ 19.9 - 43), respectively. The probability that the caregivers reliably assessed their compression depth or chest recoil (specificity) was $87.3 \%(95 \%$ CI 76.8 - 93.7) and $79.8 \%$ (95\%CI 71.1 - 86.5), respectively (Table 2$)$.

The caregivers' accurately predicted the adequacy of the compression depth in $44.6 \%$ (95\%CI 36.6 - 53.3) of the cases, and the adequacy of the chest recoil in $66.4 \%(95 \% \mathrm{CI}$ $57.8-74.1)$.

\section{Discussion}

This simulation-based study has been designed to compare healthcare providers' subjective assessment of chest compression quality vs objectively measured data. The current results have shown that there is a tendency to overestimate the depth of the thoracic compressions, which may lead to lower quality CPR. The healthcare provider's perception is a criterion that presents low sensitivity, although moderate specificity, of the actual performance of the chest compres- sion depth and recoil during CPR.

In real world practice, objective feedback is often unavailable during cardiac arrest scenarios. The current research demonstrates that providers may be overestimating the quality of compressions leading to lower quality CPR with potentially negative effects on outcomes.

These findings are consistent with those of other reports that have detected deficiencies in chest compression quality in healthcare professionals [3]. Previous clinical simulation-based experiences have also shown that experienced CPR instructors cannot accurately assess the quality of chest compression depth [4]. The use of real-time CPR feedback mechanisms allows for detecting performance mistakes, which can substantially improve the quality of the technique [5-8]. Training with CPR simulators allows for better evaluation of quality parameters in a standardized model for CPR training and skill improvement.

This study has several limitations. First, our observations were limited to 2 minutes of hands-only CPR, so our results show the performance of providers without physical fatigue. Second, in our simulation we controlled the compression rate with a metronome to mimic the real world presence of metronomes on defribillators, which may affect the results [9]. Finally, despite our efforts to produce high-quality and highly realistic simulation spaces, the use of manikins (although common in training and assessment of clinical skills) does not fully reflect the clinical scenario of a cardiac arrest in humans. A compression measured by Resusci Anne may not correlate with a clinically effective compression, and the rescuer's attitude during a simulated situation may differ from an actual cardiac arrest.

In summary, our study has demonstrated that there is a disconnect between the quality of compression being 
delivered during CPR and the providers perception of the quality; and that more objective methods to assess the quality of compressions during CPR should be considered. This may impact patient outcomes during cardiac arrest.

It can be concluded that a more objective method to assess the quality of compressions during CPR should be considered.

\section{ACKNOWLEDGEMENTS}

The authors would like to thank and acknowledge the contributions of the following individuals, who assisted with data collection: Ander Herreros-Casado and Eider DíezZaballa.

\section{CONFLICT OF INTEREST}

All authors declare that they have no conflicts of interest.

\section{FUNDING INFORMATION}

This work was funded by the Bilbao-Basurto Integrated Healthcare Organisation (Osakidetza - Basque Health Service).

\section{REFERENCES}

[1] Perkins GD, Handley AJ, Koster RW, Castrén M, Smyth MA, Olasveengen $\mathrm{T}$ et al. European resuscitation council guidelines for resuscitation 2015: section 2. Adult basic life support and automated external defibrillation. Resuscitation. 2015;95:81-99.

[2] Meaney PA, Bobrow BJ, Mancini ME, Christenson J, de Caen AR, Bhanji $\mathrm{F}$ et al. Cardiopulmonary resuscitation quality: improving cardiac resuscitation outcomes both inside and outside the hospital: a consensus statement from the American Heart Association. Circulation. 2013;128:417-35.

[3] Aufderheide TP, Pirrallo RG, Yannopoulos D, Klein JP, von Briesen $\mathrm{C}$, Sparks $\mathrm{CW}$ et al. Incomplete chest wall decompression: a clinical evaluation of CPR performance by EMS personnel and assessment of alternative manual chest compression-decompression techniques. Resuscitation. 2005;64:353-62.

[4] Brennan EE, McGraw RC, Brooks SC. Accuracy of instructor assessment of chest compression quality during simulated resuscitation. CJEM 2016;18:276-82.

[5] Wang JC, Tsai SH, Chen YH, Chen YL, Chu SJ, Liao WI. Kinectbased real-time audiovisual feedback device improves cardiopulmonary resuscitation quality of lower-body-weight rescuers. Am J Emerg Med. 2018;36:577-82.

[6] Calvo-Bueya JA, Calvo-Marcos D, Marcos-Camina RM. Estudio aleatorizado de la relación entre el uso del dispositivo CPRmeter ${ }^{\circledR}$ y la calidad de las compresiones torácicas en una resucitación cardiopulmonar simulada. Enferm Intensiva. 2016;27:13-21.

[7] Cheng A, Overly F, Kessler D, Nadkarni VM, Lin Y, Doan Q et al. Perception of CPR quality: Influence of CPR feedback, Just-in-Time CPR training and provider role. Resuscitation. 2015;87:44-50.

${ }^{[8]}$ Bobrow BJ, Vadeboncoeur TF, Stolz U, Silver AE, Tobin JM, Crawford SA et al. The influence of scenario-based training and real-time audiovisual feedback on out-of-hospital cardiopulmonary resuscitation quality and survival from out-of-hospital cardiac arrest. Ann Emerg Med. 2013;62:47-56.

[9] González-Otero DM, Russell JK, Ruiz JM, Ruiz de Gauna S, Gutiérrez JJ, Leturiondo LA et al. Association of chest compression and recoil velocities with depth and rate in manual cardiopulmonary resuscitation. Resuscitation. 2019;142:119-26.

How to cite this article: Sendoa Ballesteros-Peña, Gorka VallejoDe la Hoz, Irrintzi Fernández-Aedo, Alejandro Etayo-Sancho, Leire Berasaluze-Sanz, Josune Domínguez-García. Accuracy of Healthcare Providers'Perception of Chest Compression Depth and Chest Recoil. Signa Vitae. 2020;16(1):159-162. doi:10.22514/sv.2020.16.0021. 